\title{
Spatio-Temporal Pain Estimation Network with Measuring Pseudo Heart Rate Gain
}

\author{
Dong Huang, Xiaoyi Feng, Haixi Zhang, Zitong Yu, Jinye Peng, Guoying Zhao, Senior Member, IEEE, \\ Zhaoqiang Xia, Member, IEEE
}

\begin{abstract}
Pain is a significant indicator that shows people are suffering from an unwell experience and its automatic estimation has attracted much interest in recent years. Of late, most estimation methods are designed to capture the dynamic pain information from visual signals while a few physiological-signal based methods can provide extra potential cues to analyze the pain more accurately. However, it is still challenging to capture the physiological data from patients as it requires contact devices and patients' cooperation. In this paper, we propose to leverage the pseudo physiological information by generating new modal data from the original visual videos and jointly estimating the pain by an end-to-end network. To extract the representations from bi-modal data, we design a spatio-temporal pain estimation network, which employs a dual-branch framework for extracting pain-aware visual and pseudo physiological features separately and fuses the features in a probabilistic way. The inherent vital sign, i.e., heart rate gain (HRG), from pseudo physiological information can be utilized as an auxiliary signal and integrated with the visual pain estimation framework. Moreover, speciallydesigned 3D convolution filters and attention structures are employed to extract spatio-temporal features for both branches. To use the HRG as an auxiliary way for pain estimation, we propose a probabilistic inference model by jointly considering the visual branch and physiological branch, which makes our model estimate the pain comprehensively. Experiments on two publicly-available datasets show the effectiveness of introducing the pseudo modality, and the proposed method can outperform the state-of-the-art methods.
\end{abstract}

Index Terms-Pain Estimation, Pseudo Modality, Spatiotemporal network, 3D Convolution and Attention, Probabilistic Inference.

\section{INTRODUCTION}

$\mathbf{P}$ AIN is a complex physiological and psychological activity, which is one of the most common clinical symptoms [1]. When the body feels pain, it is actually a warning that the body is harmed, causing a series of defensive and protective reactions [2]. Pain is one of the key signs for patients to receive treatments. When patients feel unbearable pain, medical institutions need to adopt corresponding treatment or analgesia programs. Conversely, when patients feel mild or no

D. Huang, X. Feng, and Z. Xia are with School of Electronics and Information, Northwestern Polytechnical University, Xi'an 710129, China. (Corresponding author: Zhaoqiang Xia, e-mail: zxia@nwpu.edu.cn)

H. Zhang is with College of Information Engineering, Northwest A\&F University, Yangling 712100, China.

Z. Yu is with Center for Machine Vision and Signal Analysis, University of Oulu, 90014 Oulu, Finland.

J. Peng is with School of Information Science and Technology, Northwest University, Xi'an 710127, China.

G. Zhao is with School of Information Science and Technology, Northwest University, Xi' an 710127, China, and with Center for Machine Vision and Signal Analysis, University of Oulu, 90014 Oulu, Finland. pain, that indicates they are in a better condition of treatment [3]. Currently, the practical method for pain estimation mainly depends on the patient's self-report, i.e., dictating the pain intensity from 0 to 10 levels within a specified time [4]. Although this method improves the hospitalization experience of patients, it is not effective for vulnerable groups, who cannot accurately describe their pain and other symptoms. These groups include 1) infants, toddlers, and children; 2) adults with cognitive impairment such as advanced dementia; 3) people with intellectual disability; 4) critically ill or unconscious people; and 5) people who are terminally ill [5]. Also, owing to the subjectivity of self-report, this method may lead medical staff to make an incorrect judgement about the patient's conditions. Therefore, the automatic estimation method has attracted interests in the realm of patient monitoring.

To capture or describe facial muscle movements caused by pain, some visual methods are firstly proposed. In these works, there are several earlier approaches that estimate pain intensity from static face images. They employed handcrafted features [6], [7], [8] or deep networks [9], [10] to detect pain or estimate the intensity. To further estimate the pain intensity more accurately, some researchers expected to use dynamic features in video sequences. Because of this, many traditional techniques [11], [12] and deep learning methods [13], [14], [15] were proposed to capture the temporal information of pain videos. However, the aforementioned methods mainly involve the visual faces and cannot leverage other cues. To explore more information from multiple views, a few approaches began to use multi-modal data for estimating pain intensity [16], [17], [18]. The extra modal data (e.g., physiological modality) uses multiple modalities to complement each other and further improve the performance for pain estimation. But the extra modality (e.g., physiological-modal data) requires the contact devices and the patients' cooperation for collection environment. This would limit the application of automatic pain estimation in real world.

Therefore, obtaining extra modal data without using contact equipment and patients' cooperation would be advantages. Inspired by the recent success of estimating the physiological signals remotely from videos [19], [20], it becomes possible to generate an extra modality from only visual face data, which requires no special equipment but only cameras. On the other hand, when patients have experiences of pain, their heart rate would have corresponding changes [21], [22], which can be used as a cue for pain estimation. Based on this, we propose to generate a pseudo physiological signal (i.e., heart rate gain) while jointly considering the facial pain movements 
and physiological-signal variation for pain estimation. The existing heart rate estimation approaches could be used to extract the heart rate and then calculate the statistic features for pain estimation. However, there is no specific method to use this physiological information to help extract facial pain features. Consequently, how to use the extra modal data and fuse bi-modal data still needs to be further investigated.

Therefore, we design a dual-branch deep network for pain estimation by extracting pain-aware features visually and physiologically and fusing them in a probabilistic way for joint estimation. In this framework, we firstly design a visual branch and then incorporate a physiological branch for pseudo heart rate gain (HRG) generation as an auxiliary stream. Furthermore, to extract the spatio-temporal features from two streams simultaneously, we employ specially-designed 3D convolution filters (i.e., $\mathrm{R}(2+1) \mathrm{D})$ and attention structures that ensure the network could analyze dynamic information from video sequences and sense pain-aware regional changes. In order to explore physiological and visual streams jointly, we devise a differentiable probabilistic inference model called the HRG analyzer, which employs the prior information to provide a probability distribution of pain intensity according to the corresponding relationship between HRG and pain intensity. This probability distribution is finally fused with visual inference to the pain estimation. The main technical contributions of this work are summarized:

- We propose to generate a pseudo physiological signal and correspondingly design a dual-branch framework for pain estimation from two perspectives (one for visual features and the other for pseudo physiological features). Extensive experiments are performed to show this framework achieves competitive performance.

- The special 3D convolution filters and attention structures are employed to capture the spatio-temporal features, which models the pain-aware dynamic deformations and influences of different facial regions.

- We devise a differentiable probabilistic inference model with prior information for durative back-propagation, which can jointly fuse the visual and pseudo physiological features.

\section{RELATED WORK}

Over the past decade, various techniques for pain estimation have been continuously explored. According to the modality of input data, these approaches can be roughly divided into two categories: visual methods and multi-modal methods. Below we briefly discuss them separately.

\section{A. Visual Methods}

For visual face estimation methods, early researchers tended to detect pain or estimate intensity from a static face image. For instance, in [6], they extracted Gabor features, independent components and flow-based features, which trained support vector machine (SVM) classifiers to distinguish the real pain and the posed pain. In [7], active appearance models were employed to decouple shape and appearance features which were classified by SVM. Following this research, Lucey et al.
[23] used the same method to give some baseline results on the UNBC dataset. For diminishing the subjects differences, Rudovic et al. [24] employed the heteroscedastic conditional ordinal random field to propose an Action Units (AUs) intensity estimation model adapted to individual changes. In the work of [8], Florea et al. tried histograms of topographical features to generalize pain texture features. In order to alleviate the pain estimation problem, Wang et al. [9] fine-tuned a face verification network to generate a deep network which employed a regularized regression loss. Yang et al. [10] proposed a feature representation, which encoded traditional descriptors and powerful deep features by a weighted process, to form an efficient representation of facial images.

To explore more temporal information, some researchers expected to estimate pain intensities from facial video sequences. They believed that pain is a dynamic process involving onset, apex, and offset. As stated in [25], a steerable and separable filter was used to measure the energy released by the facial muscles. These measured results were employed as a representative feature of pain to detect pain and estimate pain intensity. In [26], Zhao et al. focussed more on the mechanism of machine learning. An efficient optimization algorithm based on alternating direction method of multipliers was proposed. Then, they used their methods to predict pain intensities under fully supervised, weakly supervised, and unsupervised conditions. Werner et al. [11] analyzed the relative positional changes due to the movement of some points on faces during the pain producing process. For this reason, facial activity descriptors were proposed to describe the pain. As for selfreported pain, Liu et al. [12] employed some handcrafted personal features and multi-task learning to personalize pain intensities. Zhou et al. [13] applied recurrent convolutional neural network (RCNN) architecture to represent dynamic pain features and regressed the continuous pain intensity. As stated by [14], they dealt with the pain estimation task by using the combination of convolutional neural networks (CNNs) and long short-term memory (LSTM). In their work, a pre-trained CNN model was used to fine tune on the pain dataset, and LSTM was used to explore the dynamic changes of $\mathrm{CNN}$ features to represent the temporal facial changes of video sequences. Owing to the temporal expression ability of LSTM, they obtained better performance than RCNN. Similar to [12], Martinez et al. [27] used LSTM to estimate pain intensity in video sequences while the hidden conditional random field (HCRF) was used to output the visual analog scales (VAS) of each subject. In [15], CNN features were aggregated by exploiting statistical information, which was used to represent the facial changes of the videos. According to [28], a residual 3D deep model was presented for dynamic spatio-temporal features of faces in video sequences. They proposed a knowledge transfer architecture from a $2 \mathrm{D}$ pretrained model for training this 3D deep model. Because of these special techniques for pain estimation, they obtained better performance than previous methods. Different from this residual 3D network, in this context, we employ a more effective $3 \mathrm{D}$ convolution filter as well as attention structure, which has different architecture and operations.

With these specially-designed features, these automatic pain 
estimation systems begin to outperform the humans and achieve good performances for wide applications without using any special equipment. However, there is still room to promote pain estimation as these approaches only use visual features directly without considering other modalities.

\section{B. Multi-modal Methods}

To provide the information for more accurate emotion perception, some researchers have explored the possibility from other modal signals, i.e., multi-modal methods. For example, Tzirakis et al. [29] proposed an emotion recognition system involving audio and visual face modalities. To capture the emotional content for various styles of speaking, they employed a 1D CNN to extract features from the speech, while a deep residual network was used for the facial video. In their work, LSTM was used to capture the contextual information of fused features. Since it is believed that physiological signals will change according to emotional responses [30], the physiological signals also become the primary supplement to visual face modality. As stated in [31], they combined EEG and eye movements for integrating the internal cognitive states and external subconscious behaviors of users to improve the emotion recognition accuracy.

Similar to general emotion perception, facial pain also follows the human body's protective mechanism, which causes physiological signals to change[32]. Inspired by this, several approaches have explored multi-modal information with the contact devices for pain estimation and achieved more accurate performance. In [18], various physiological signals were utilized to recognize self-report pain by a multi-task network, which achieved competitive performances compared to only using facial information. Moreover, other methods began to explore multiple modalities jointly. For instance, Hammal et al. [33] combined facial features, sensor signals and environmental background as dynamic pain features. Similarly, Werner et al. [16] extracted dynamic spatio-temporal features not only from facial expression and head movement but also from involved physiological signals, such as galvanic skin response (GSR), electromyography (EMG) and electrocardiogram (ECG). Their experimental results showed that the combination of multiple modalities is significantly better than a single modality. Besides, it can also be concluded from these results that facial expressions and physiological signals contributed more to pain estimation. In [17], Kächele et al. utilized multi-modal features which consisted of facial expression features, head pose features and bio-physiological features for pain intensity estimation. They also reached similar conclusions from their experiments. For the continuous estimation of pain intensity, Kächele et al. [34] further investigated various ways to estimate the similarity of persons and to retrieve the most informative ones using meta information, personality traits, and machine learning techniques. In [35], the deep denoising convolutional autoencoders were presented for the classification of pain intensities based on multiple physiological signals.

As can be seen, if there are more available signals, researchers can explore more information for pain estimation.
As the facial muscle changes are only one aspect of the pain response, pain is also manifested in various physiological signals [18]. However, for these multi-modal methods, the contact devices for receiving physiological signals and the cooperation of patients for collection environment are always necessary in the real world, which would greatly limit the application of such methods. Besides, the semantic alignment of multi-modal data is also challenging as multiple devices (e.g., electrocardiogram monitor and camera) are employed. To break this limit, we propose to leverage non-contact physiological information implicitly for the videos and design an endto-end pain estimation model for extracting bi-modal features.

\section{MethodolOGY}

In this section, we present our proposed deep method that estimates pain intensity from videos in two views (i.e., visually and physiologically). The pipeline of the proposed method is shown in Fig. 1. We will successively introduce three procedures, including data preprocessing, deep network and parameter learning. After the video sequences are preprocessed, they are fed into a dual-branch network to extract dynamic pain-aware features. The visual branch provides the visual features from the facial regions while the physiological branch outputs the heart rate gain (HRG) as the representation of pseudo physiological modality. Furthermore, an HRG analyzer is implemented to connect HRG with the pain intensity, and the probability inference would integrate HRG with the visual branch for final estimation. Multiple losses towards different tasks (i.e., pseudo HRG generation and pain estimation) are integrated together to perform two-stage model learning.

\section{A. Preprocessing}

Before feeding the pain videos into the deep model for extracting bi-modal features, it is necessary to preprocess the raw video data. Our entire preprocessing procedure is shown in Fig. 2, which mainly includes face cropping, key frames selection and data augmentation.

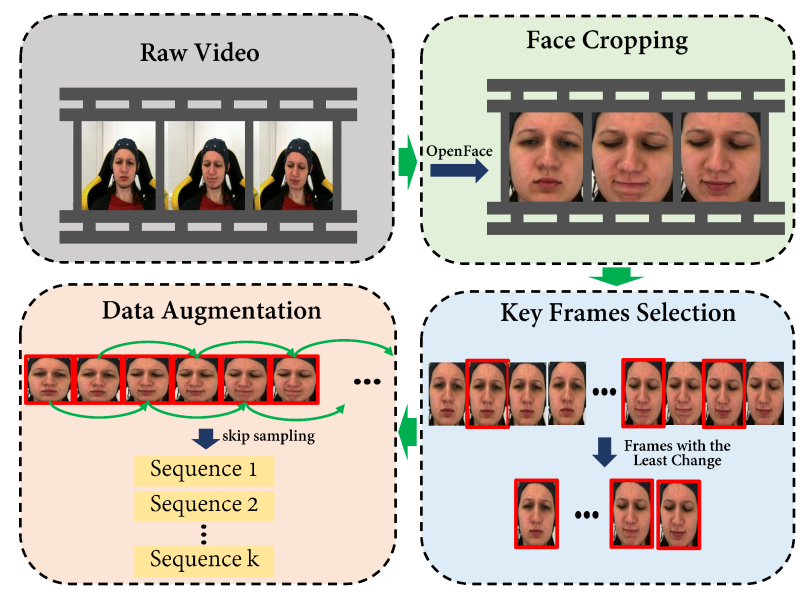

Fig. 2. The preprocessing procedure for video sequences.

Face Cropping. Since the most direct response to pain is the local regional deformation of the face [14], the main 


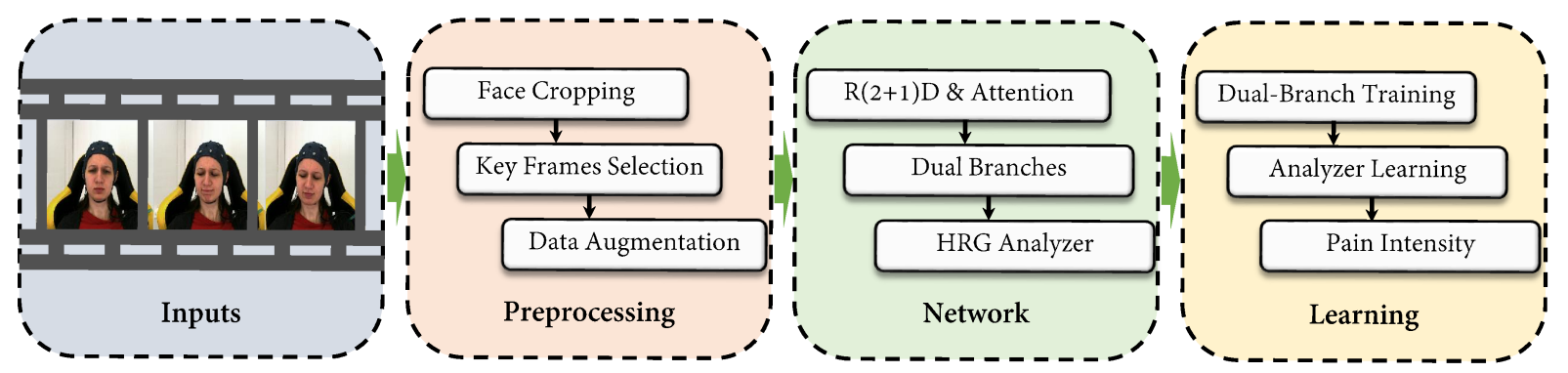

Fig. 1. The pipeline of the proposed method.

interest is the facial region. To go along with this, remote physiological vital signs could be measured from facial skin regions [36]. Therefore, before feeding to the network, the background information needs to be removed. We employ OpenFace [37] to segment the facial regions from the original images. OpenFace is a robust face detector which can process the images with non-frontal and little occluded faces or with poor illumination conditions. It accomplishes the detection based on multi-task CNNs. OpenFace is capable of aligning and cropping facial regions from videos. Therefore, this tool can meet the requirements of our method.

Key Frames Selection. Another task is how to pick out several image frames from the video sequences to reduce redundancy. Since not all these images contain pain expressions, only the image frames with face regional changes could represent the pain intensity. So there is no need to feed the entire video into the network, resulting in the redundancy of network structure. Here, we refer to the method of [38] to select the key frames of videos. In a time window of $2 i$ frames, where $i$ is set to 3 empirically, the histograms of frames are calculated. A chi-square distance is employed to measure the difference of successive frame histograms. The frame with the least difference is picked up as the key frame in this time window. This method could select the most representative frame in a time window for video sequence.

Data Augmentation. In addition, since a deep network is used for pain estimation, massive data are required for model training. However, the number of videos in public datasets is limited as the collection of pain videos is time-consuming. To address this problem, we decide to sample a video multiple times to cope with deep network training. Specifically, we perform frame skip sampling on a processed video that only contains key frames. In this way, we could process one video sequence into several training samples.

\section{B. Bi-Branch Deep Network}

1) Network Architecture: To explore spontaneous spatiotemporal variation in human faces, we aim to analyze the videos from a new physiological perspective, which is measured remotely from visual videos. According to the psychology knowledge, we know that the heart rate changes when a person feels pain and these two indicators (i.e., pain and heart rate) are highly related [21], [22]. Thus, we choose to explore the relationship of pain and heart rate by measuring the signal gain. Here, the signal gain is defined as the increment degree of heart rate relative to the one in the painless condition. This is therefore called heart rate gain (HRG), which can be represented by a single value. Then we can use the HRG to estimate the pain intensity. Different from the physiological indicators with physical devices, we generate the pseudo signal without relying on any physical measurement devices, which can be more easily applied in real-world environments. To this end, we propose a dual-branch network for estimating pain intensity only from videos, which is shown in Fig. 3.

In our network, there are three subsections: 1) the shared blocks and special structures, 2) visual and physiological branches, and 3) modality fusion and pain estimation. At the beginning, several layers are employed to extract spatiotemporal dynamics and explore a potential space for subsequent dual-branch processing. Apart from the facial movements feature extraction, we tend to employ this network to generate pseudo HRG and further extract physiological features. Therefore, this deep network consists of two branches (one for visual pain features, and the other for pseudo physiological features). The same structure is selected for these two branches, which could use similar filter sizes shown in Table I. Following the physiological branch, an HRG analyzer is used to map the HRG value to the probability of pain intensities. The probabilistic fusion inference is implemented in this analyzer. The final pain intensity estimation will fully consider the influence of pseudo physiological modality and visual modality, which results in a comprehensive pain estimation. In the following, we will introduce these procedures in details.

TABLE I

THE CONFIGURATION OF SPATIO-TEMPORAL PAIN ESTIMATION NETWORK WITH MEASURING PSEUDO HEART RATE GAIN.

\begin{tabular}{|c|c|c|c|}
\hline layers & output size & filters \\
\hline & & \multicolumn{2}{|c|}{ Shared Blocks } \\
\hline Conv3D & $\mathrm{L} \times 56 \times 56$ & \multicolumn{2}{|c|}{$1 \times 1 \times 1,64$} \\
\hline $\mathrm{R}(2+1) \mathrm{D}$ & $\mathrm{L} \times 56 \times 56$ & {$\left[\begin{array}{l}3 \times 3 \times 1,64 \\
1 \times 1 \times 3,64\end{array}\right] \times 2$} \\
\hline $\mathrm{R}(2+1) \mathrm{D}$ & $\frac{L}{2} \times 28 \times 28$ & {$\left[\begin{array}{l}3 \times 3 \times 1,128 \\
1 \times 1 \times 3,128\end{array}\right] \times 2$} \\
\hline $\mathrm{R}(2+1) \mathrm{D}$ & $\frac{L}{4} \times 14 \times 14$ & {$\left[\begin{array}{l}3 \times 3 \times 1,256 \\
1 \times 1 \times 3,256\end{array}\right] \times 2$} \\
\hline & & Visual Branch & Physiological Branch \\
\hline $\mathrm{R}(2+1) \mathrm{D}$ & $\frac{L}{4} \times 14 \times 14$ & {$\left[\begin{array}{l}3 \times 3 \times 1,256 \\
1 \times 1 \times 3,256\end{array}\right] \times 2$} & {$\left[\begin{array}{l}3 \times 3 \times 1,256 \\
1 \times 1 \times 3,256\end{array}\right] \times 2$} \\
\hline $\mathrm{Attention}$ & $\frac{L}{4} \times 14 \times 14$ & - & - \\
\hline $\mathrm{R}(2+1) \mathrm{D}$ & $\frac{L}{8} \times 7 \times 7$ & {$\left[\begin{array}{c}3 \times 3 \times 1,512 \\
1 \times 1 \times 3,512\end{array}\right] \times 2$} & {$\left[\begin{array}{l}3 \times 3 \times 1,512 \\
1 \times 1 \times 3,512\end{array}\right] \times 2$} \\
\hline & $1 \times 1 \times 1$ & spatio-temporal pooling & spatio-temporal pooling \\
\hline $\mathrm{fc}$ & $1 \times 1 \times 1$ & \multicolumn{2}{|c|}{-} \\
\hline & & \multicolumn{2}{|c|}{ Modalities Fusion } \\
\hline
\end{tabular}




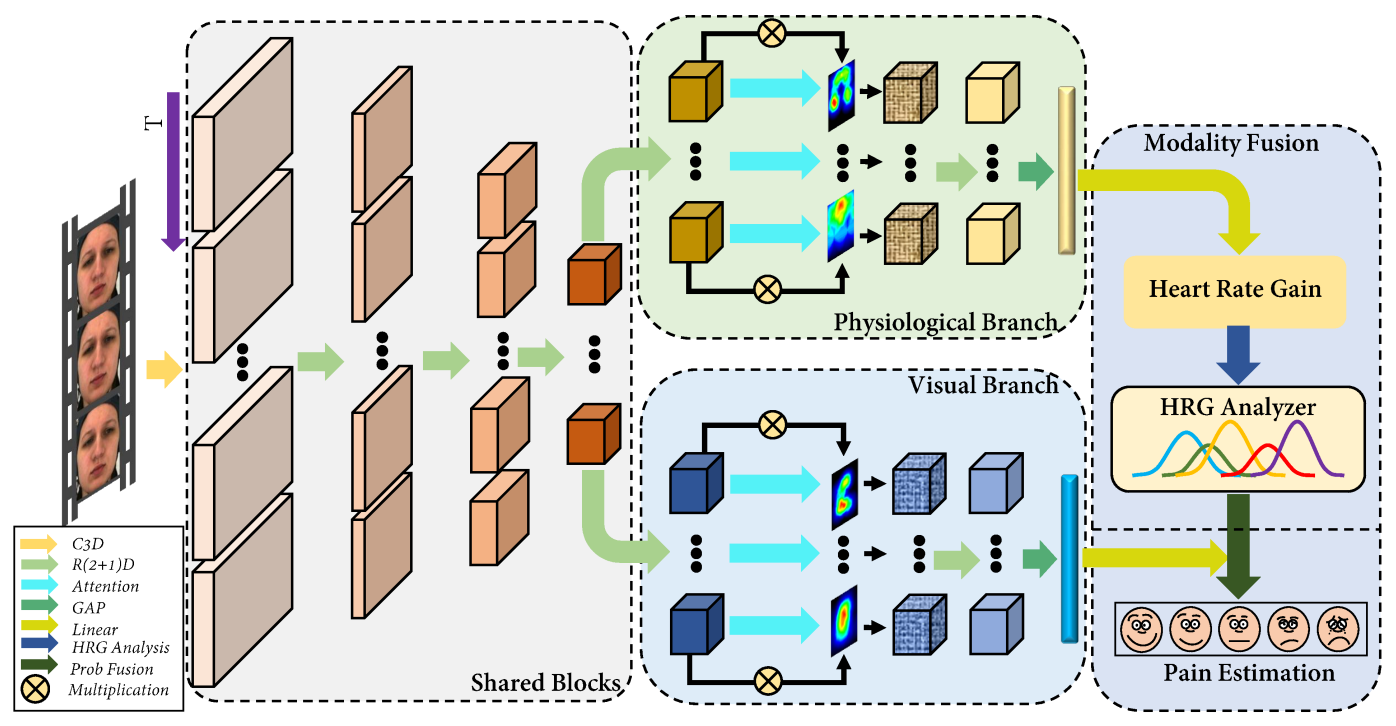

Fig. 3. The architecture of deep pain estimation network with measuring heart rate gain. There are three parts: the shared blocks, visual and physiological branches, and modality fusion.

2) Shared Blocks and Special Structures: Shared Blocks. Since the training samples for the pain estimation task are not very large, we employ several shared blocks and operations to reduce the parameters of deep models for saving the computational cost [39], [40]. The shared blocks with several layers are used to project the selected frames into a potential space and further used to extract features by two branches with shared architecture but different parameters.

3D Convolution. Furthermore, in these shared blocks and subsequent branches, we decide to use $3 \mathrm{D} \mathrm{CNN}$ to extract features. However, the training of conventional 3D CNN from scratch results in expensive computational cost and memory demand. So in order to speed up the computation, the 3D convolution is approximated by a $2 \mathrm{D}$ convolution followed by a 1D convolution [41]. This structure can introduce an additional nonlinear rectification between these two convolutions thus making it easier to optimize. Therefore, we use a “(2+1)D" convolutional block, which explicitly factorizes 3D convolution into two separate and successive operations: a 2D spatial convolution and a 1D temporal convolution. We employ the residual structure for this $(2+1) \mathrm{D}$ block, which is denoted as $\mathbf{R}(\mathbf{2 + 1}) \mathbf{D}$. In this structure, $N_{i} 3 \mathrm{D}$ convolutional filter of size $N_{i} \times t \times d \times d$ is substituted with a (2+1)D block consisting of $N_{i}$ 2D convolutional filters of size $N_{i} \times 1 \times d \times d$ and $M_{i}$ temporal convolutional filters of size $M_{i} \times 1 \times 1 \times 1$. The parameter $M_{i}$ determines the dimensionality of the intermediate subspace where the data is projected between the spatial and temporal convolutions. $M_{i}$ is set as $M_{i}=\left\lfloor\frac{t d^{2} N_{i-1} N_{i}}{d^{2} N_{i-1}+t N_{i}}\right\rfloor$. In this way, the number of parameters in the (2+1)D block is approximately equal to that in full 3D convolution. This decomposition can be used as a substitute for any 3D convolution filter.

Attention Structure. To capture the features for pain estimation and enable the ability of distinguishing subtle changes [42], [43], we further propose an attention structure for the basic $\mathrm{R}(2+1) \mathrm{D}$ convolution, which is used in two branches respectively. This attention structure allows the network to im- prove the importance of certain important dimensions of input features and to decrease the unrelated dimensions spatially and temporally. This structure is a self-attention mechanism that extracts the attention map from its own feature map. In detail, $X \in \Re^{T \times W \times H \times D}$ is the input feature maps and $Y \in \Re^{T \times W \times H \times D}$ is the output of this attention structure. Then, $Y$ is computed as follows:

$$
Y=\sigma\left(W_{2}\left(\tanh \left(W_{1} X+b_{1}\right)\right)+b_{2}\right) \odot X
$$

where $\odot$ denotes the multiplication in feature spatial and temporal dimensions. Since we extract different attention maps for different feature maps, the attention map could change over the different frames. In this way, we realize attention structure by the attention map $\sigma\left(W_{2}\left(\tanh \left(W_{1} X+b_{1}\right)\right)+b_{2}\right)$ multiplication operation. It is noted that this attention structure could be added after any layer of 3D network.

3) Visual and Physiological Branches: Following the shared blocks, two extra branches are included to extract the visual and pseudo physiological features separately, which are called visual branch and physiological branch. As shown in Table I, these two branches employ the same architecture, which has one attention structure, one 3D convolution layer, one global pooling and one fully-connected layer. Although the architectures in the two branches are the same, they usually do not learn the same parameters as they focus on different modalities. The basis of the HRG generation in videos is to capture the light scattering changes of the skin in ROIs [44], whereas pain estimation shows different ROIs which show texture changes around the eyes and mouth [45]. For this reason, as shown in Fig. 3, we achieve different attention maps when using attention structure. This makes our network focus on the changes of their corresponding important regions in different branches. The convolutional layers after the attention structure also use individual parameters to obtain their own features. This way ensures the relevance of features and distinguishes their differences. Finally, the last fully-connected 
layers in these two branches output the HRG value $H$ and the visual estimation probability $P_{v}$, respectively, which would be fused in a later stage for final pain estimation.

To learn the parameters of two branches more efficiently, we choose to train these two branches separately and then fine tune the entire model jointly, for which more details would be introduced in Section III-C. Here, the generation of pseudo labels for the physiological branch will be explained firstly. We provide two types of generating HRG annotations from the metadata of datasets. The first one is based on physiological signals, which can be used for the datasets having ECG signals related to HRG. This way obtains the gain value from the ECG signal as shown in Fig. 4. It is worth noting that the heart rate changes over the various research subjects. We propose to use HRG to represent the relative heart rate increase, which means the signal gain of heart rate in a video sequence compared to the average heart rate in painless video sequences. The HRG value is obtained by $H R G=\frac{H R}{\text { mean }\left(H R_{(P I=0)}\right)}$, where $P I=0$ means pain intensity is zero. Then, these values are normalized to $[0,1]$. This way could remove the effects of different heart rates caused by different individuals. The second type is based on the published methods to measure heart rate from face video analysis, which is used in the datasets that do not provide any physiological signals. In this case, we leverage the existing heart rate estimation model [19] to obtain heart rate, and then the HRG value could be obtained in the same way.

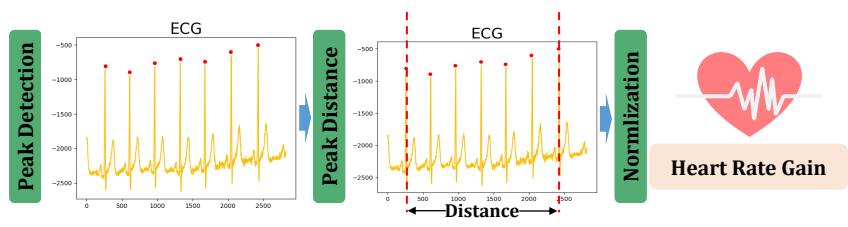

Fig. 4. The steps to obtain HRG from ECG signals.

4) Modality Fusion and Pain Estimation: Since the outputs of two branches in the dual-branch network above have different representations, their outputs cannot be easily fused together for pain estimation [46]. To further use the physiological HRG for the visual branch, we firstly explore the inherent correspondences between HRG value and pain and then fuse them in a probabilistic way. Following the physiological branch, we design an HRG analyzer to provide the quantitative relationship between HRG and pain, which can connect the HRG values to pain levels.

With the generation of pain sensation, the heart rate changes accordingly. The more severe the pain, the more the heart rate increases (the greater the HRG becomes). So there is a strong relationship between pain and HRG [21], [22]. Actually, the HRG value $H$ is a continuous variable while the estimated pain intensity $P I$ is a discrete variable, containing $n$ levels. The question now is how to map a continuous variable $H$ to a discrete variable $P I$. In general, the $H R G$ value in each pain level is not evenly distributed. Due to the diversity of samples, $H$ mostly concentrates near the mean. The farther away from the mean, the less frequent $H$ occurs. Besides, the greater the variance of $H$, the more widely it distributes, while the smaller the variance corresponds the more concentrated distribution.
This is roughly matched with Gaussian distribution, which can be used to model the relationship between HRG and pain.

Assuming in a certain pain intensity, the variable $H$ follows a Gaussian distribution, $H \sim G\left(\mu, \sigma^{2}\right)$, where $\mu$ and $\sigma$ denote the mean and variance of $H$ in a specific pain intensity. For each pain intensity, the $\mu$ and $\sigma$ of $H$ could be easily obtained by statistics. Under this condition, if a specific HRG value $h$ has been estimated from a video, we could compute the probability $P_{r}$ when pain intensity $P I=p_{i}$ in the following equation:

$$
P_{r}\left(H=h \mid P I=p_{i}\right)=\frac{1}{\sqrt{2 \pi} \sigma_{i}} \exp \left(-\frac{\left(h-\mu_{i}\right)^{2}}{2 \sigma_{i}^{2}}\right)
$$

where $i$ denotes the $i$-th pain level. When a subject (person) reveals a certain pain intensity $p_{i}$, this way measures the conditional probability of HRG connecting $h$ to $p_{i}$. Furthermore, according to the Bayesian theory, we obtain the posterior probability of pain level $p_{i}$ as follows

$P_{r}\left(P I=p_{i} \mid H=h\right)=\frac{P_{r}\left(P I=p_{i}\right) \cdot P_{r}\left(H=h \mid P I=p_{i}\right)}{\sum_{i} P_{r}\left(H=h \mid P I=p_{i}\right) \cdot P_{r}\left(P I=p_{i}\right)}$

Since the sample of each pain intensity level has the same probability in the training set and real world, $P_{r}\left(P I=p_{i}\right)$ obeys the uniform distribution, which has the same value for all levels. So the formula above can be further simplified to the following format:

$$
P_{h_{i}}\left(P I=p_{i} \mid H=h\right)=\frac{P_{r}\left(H=h \mid P I=p_{i}\right)}{\sum_{i} P_{r}\left(H=h \mid P I=p_{i}\right)}
$$

where $P_{h_{i}}$ means the calculated probability from HRG value for pain intensity $P I=p_{i}$. Following Eq. 4, the probabilities of HRG in various pain intensities are able to be calculated. If there are several pain intensities that need to be estimated, their HRG distributions in these pain intensities can also be counted, which is illustrated in Figs. 12 and 13. Notably, these distributions are both counted from the training set. Therefore, the model is not exposed to the ground truth during testing.

To fuse the probabilities from the visual branch and the HRG analyzer for the physiological branch, we choose to multiply two types of probabilities as the final estimation probability for pain intensities as follows:

$$
P_{f_{i}}=P_{h_{i}} \cdot P_{v_{i}}(i=1,2, \ldots, n)
$$

where $P_{h_{i}}$ and $P_{v_{i}}$ denote the estimation probability from HRG analyzer and visual branch respectively, $P_{f}$ is the final fusion result, and $i$ means the $i$-th pain intensity level.

\section{Parameter Learning}

To learn the parameters of the deep network above, it is necessary to design the corresponding loss function to measure the distance between the predicted value of the network and the ground-truth, and update the parameters of each layer in the deep neural network by back-propagation. Since different types of tasks have been contained in the dual-branch network, multiple loss functions will be contained in parameter learning. 
Since the task of HRG generation has continuous-value output, the Mean Square Error (MSE) loss function is utilized, which is shown in Eq. 6:

$$
L_{h}=-\sum_{j=1}^{J}\left(h_{j}-h_{j}^{p}\right)^{2}
$$

where $h_{j}$ and $h_{j}^{p}$ represent HRG ground-truth value and the prediction value of the physiological branch, respectively. $J$ is the size of each mini-batch. For the task of the visual branch, the Softmax loss function is adopted as follows:

$$
L_{s}=-\frac{1}{J} \sum_{j=1}^{J} \sum_{i=1}^{I} p_{i j} \log P_{v_{i j}}
$$

where $J$ is the size of mini-batch, and $I$ is the number of pain intensities. These two losses $L_{h}$ and $L_{s}$ are used to learn the two branches separately.

To further fine-tune the entire deep model after modality fusion, we continue to employ the loss of joint probability from final estimation to update the network parameters. After we calculate the final probability $P_{f}$ in Eq. 5, we also choose the Softmax loss function to measure the loss between the final estimation and ground-truth, which is shown in Eq. 8:

$$
L_{h p}=-\frac{1}{J} \sum_{j=1}^{J} \sum_{i=1}^{I} p_{i j} \log P_{f_{i j}}
$$

where $J$ is the size of mini-batch, and $I$ is the number of pain intensities. This loss is backpropagated as follows:

$$
\frac{\partial L_{h p}}{\partial P_{f_{i j}}}=-p_{i j} \frac{1}{P_{f_{i j}}}
$$

Then, the parameters of HRG generation task are updated by:

$$
\frac{\partial P_{f_{i}}}{\partial h}=P_{v_{i}} \frac{\partial P_{h_{i}}}{\partial h}=P_{v_{i}} \frac{A_{i} \sum_{i} P_{r}\left(h \mid p_{i}\right)-P_{r}\left(h \mid p_{i}\right) \sum_{i} A_{i}}{\left(\sum_{i} P_{r}\left(h \mid p_{i}\right)\right)^{2}}
$$

where $P_{r}\left(h \mid p_{i}\right)$ denotes $P_{r}\left(H=h \mid P I=p_{i}\right) . A_{i}$ is calculated as follows:

$$
A_{i}=-\frac{h-\mu_{i}}{\sqrt{2 \pi} \sigma_{i}{ }^{3}} \exp \left(-\frac{\left(h-\mu_{i}\right)^{2}}{2 \sigma_{i}{ }^{2}}\right)
$$

For the parameters of another branch (the visual branch), the parameters are updated according to the following equation:

$$
\frac{\partial P_{f_{i}}}{\partial P_{v_{i}}}=P_{h_{i}}
$$

The derivatives of parameter values in previous layers are successively calculated according to the back propagation.

The two-stage learning procedures are summarized in Algorithm 1. Given that the physiological branch needs to be pretrained separately, the entire network is end-to-end only during the testing phase. Therefore, the proposed model provides a non-contact multi-modal pain estimation method. Besides, the network using these loss functions is differentiable, i.e., the loss between the predicted value and the ground-truth can be backpropagated to previous layers.

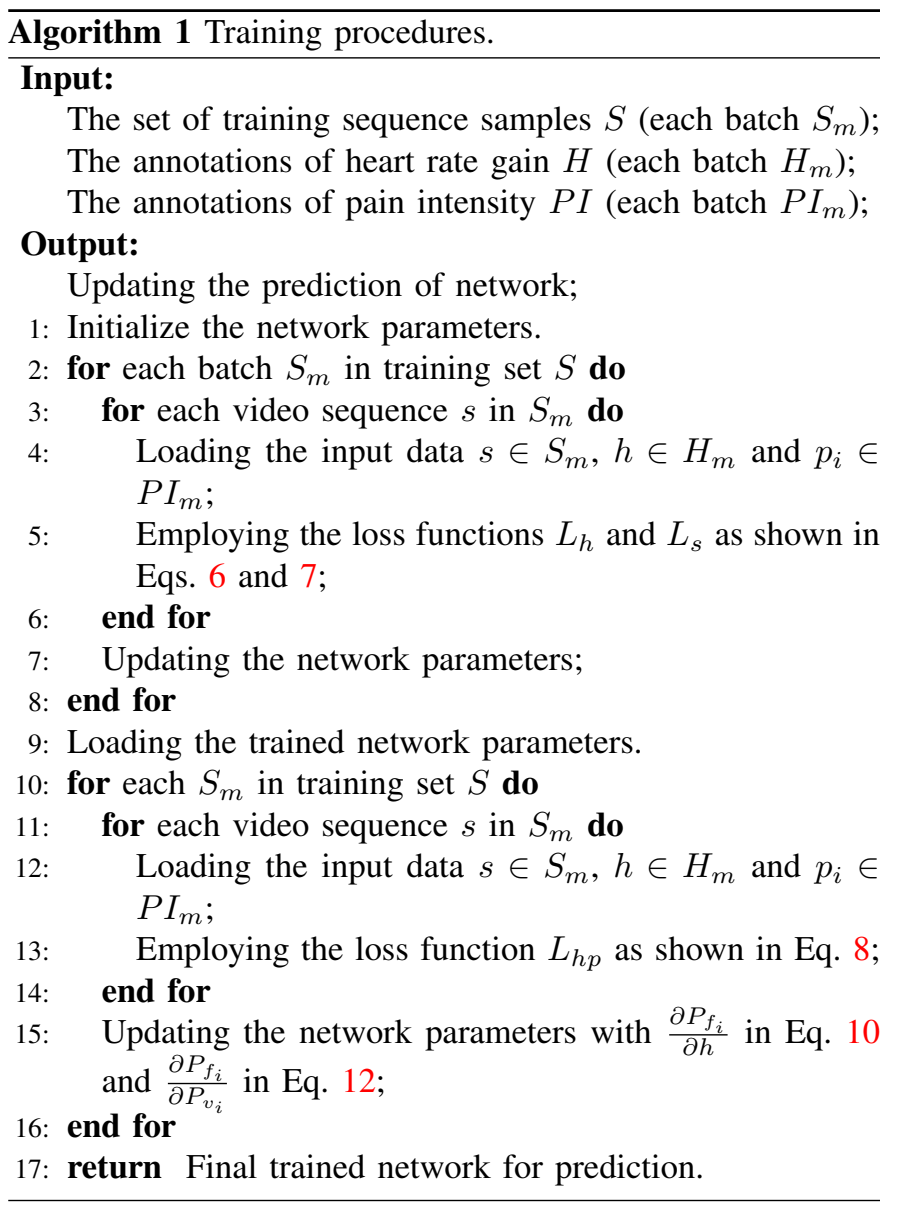

\section{EXPERIMENTS}

In this section, we conduct extensive experiments to evaluate the proposed method for pain estimation. Firstly, two publiclyavailable datasets and our implementation details are described. The effectiveness of each component in the proposed framework is then evaluated. Finally, the proposed method is compared to the state-of-the-art approaches.

\section{A. Datasets and Setups}

The BioVid [47] and Mint datasets [48] are chosen as the benchmark datasets. In the experiments, all the data are processed by the preprocessing procedure (Sec. III-A).

BioVid Dataset: A total of 90 subjects are included in this dataset, which had been recruited from three age groups. Four discrete pain intensities were induced in the right arm of each subject. Besides, some psychological signals such as the Skin Conductance Level (SCL), the electrocardiogram (ECG), the electromyography (EMG) and the electroencephalogram (EEG) were recorded. In our experiment, we only conduct the proposed method on part $\mathrm{A}$ of this dataset as shown in the first row of Fig. 5. In BioVid Part A, each of 8,700 video sequences is labeled with the respective pain stimulus intensity. So our task distinguishes a total of five discrete pain intensities: no pain (level 0), low pain (level 1, pain threshold), two intermediate intensities (level 2 and 3) and severe pain (level 4, pain tolerance). 
Mint Dataset: The Multi-modal intensity pain (Mint) dataset could also be utilized for pain estimation. A total of 20 subjects are included in this dataset. It contains a series of facial videos of subjects who are stimulated by electrical pain. Figure 5 shows some samples of this dataset in the second row. And this dataset records 9,366 various video sequences with 187,939 image frames. Each sequences is labeled with the respective electrical pain intensity. There are the same pain levels as Biovid dataset, which is also categorized into five intensities.

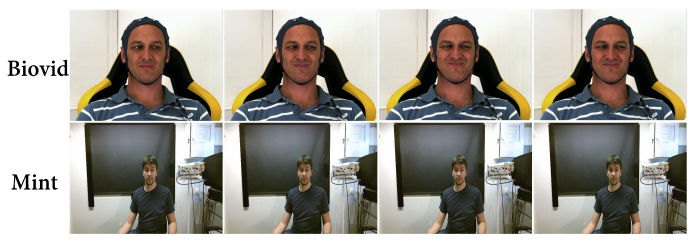

Fig. 5. Some samples of BioVid and Mint datasets.

All experiments in this context are performed in a leaveone-subject-out cross validation manner, i.e., the subjects in the testing set are excluded from the training set. Then all subjects are tested once through the cycle, and the average of the results is shown [14], [28]. The pain intensities in the datasets we used are evenly distributed, that is, the number of samples in each intensity is equal. Besides, in order to compare experimental results, the results are reported with the metrics of Accuracy on these datasets. The results are shown in a way of mean $\pm s t d$, where mean and std denote the mean and standard deviation of accuracy, respectively.

\section{B. Ablation Study and Parameter Analysis}

1) The Evaluation of $R(2+1) D$ Convolution: We first evaluate the effectiveness of convolution filter types. There are three alternatives: C3D [49], R3D [50] and R(2+1)D filters. The C3D is a kind of convolution filter with ordinary 3D convolution. The $\mathrm{R} 3 \mathrm{D}$ is the $\mathrm{C} 3 \mathrm{D}$ with residual shortcuts. The $\mathrm{R}(2+1) \mathrm{D}$ convolution used in this paper factorizes $\mathrm{R} 3 \mathrm{D}$ into two separate and successive operations, a 2D spatial convolution and a 1D temporal convolution. All of these features can be used to extract spatio-temporal features. To fairly compare each other, only the convolution kernels (i.e., $\mathrm{C} 3 \mathrm{D}$ and $\mathrm{R} 3 \mathrm{D}$ ) are replaced while other parts of the proposed network are kept. The results are shown in Table II. In the considered classes, the [all-all] means all pain intensities are classified. The others mean the classification of two intensities, for instance, [4-0] means the classification of pain intensity 4 and pain intensity 0 .

Observed from the results, $\mathrm{R}(2+1) \mathrm{D}$ convolution used in the proposed method can achieve the best performance. This is reasonable because $\mathrm{R}(2+1) \mathrm{D}$ convolution separates the spatial and temporal feature extraction process, which will add many nonlinear operations and promote the discrimination ability of the model. R3D has better performance than C3D. This suggests the network with residual shortcuts has a better feature representation ability in pain estimation. Another advantage of using $\mathrm{R}(2+1) \mathrm{D}$ convolution is that the operation of spatiotemporal decoupling is easier to optimize. The training process shown in Fig. 6 reveals that the $\mathrm{R}(2+1) \mathrm{D}$ converges faster.

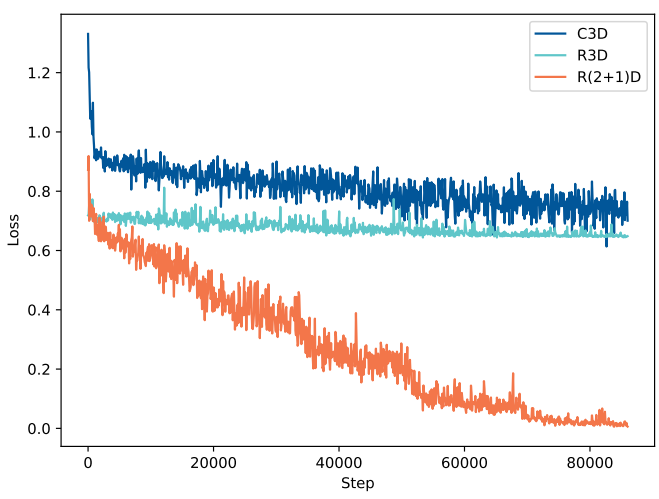

Fig. 6. The loss function decreasing of three convolution filters.

2) The Evaluation of Attention Structure: In this paper, we propose to use an attention structure to make the deep network more attentive to important regions of pain estimation, such as the regions where the texture changes of the face in the spatial domain and those frames that contain pain expressions in the temporal domain. In this section, we compare the effectiveness of this attention structure with the similar network without attention structure. The results are shown in Fig. 7, where Position $=4$ and Position $=0$ denote the network with and without attention structure, respectively. And the [all-all] means all pain intensities are classified and the [4-0] means the classification of pain intensity 4 and pain intensity 0 . As can be seen, the network with attention has better performance. One reason may be that attention structure enables the deep network to focus on the significant information. Since a video contains much redundant information, the task of pain estimation only needs to pay attention to the relevant content.

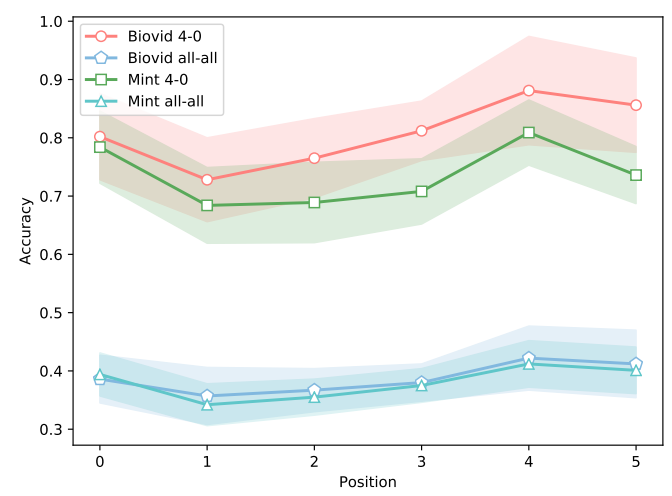

Fig. 7. The influence of attention positions. The Position $=0$ denotes there is no attention structure and the others mean the attention structure are put in different positions of the architecture.

For a more detailed analysis, we visualize the learned attention maps that are scaled to the same size of input images, which are shown in the second row of Fig. 8. Observed from heat maps, it is clear that the attention structure makes the pain-aware regions more significant, such as regions of eyes, nose and mouse. These regions are the most important manifestations of pain expression indicated in [51]. Then, we 
TABLE II

THE ACCURACY OF VARIOUS CONVOLUTION FILTERS. THREE CONVOLUTION FILTERS WERE EXPERIMENTED UNDER DIFFERENT CONDITIONS OF PAIN INTENSITY CLASSIFICATION.

\begin{tabular}{c|c|ccc|ccc}
\hline & & \multicolumn{3}{|c|}{ Biovid } & \multicolumn{3}{c}{ Mint } \\
\hline Considered Classes & Chance & C3D & R3D & R(2+1)D & C3D & R3D & R(2+1)D \\
\hline$[4-0]$ & & $78.1 \pm 5.8$ & $84.1 \pm 8.9$ & $88.1 \pm 9.3$ & $71.2 \pm 3.3$ & $74.6 \pm 2.4$ & $80.9 \pm 5.6$ \\
{$[\mathbf{4 - 1}]$} & & $70.6 \pm 11.0$ & $79.2 \pm 10.2$ & $79.9 \pm 3.7$ & $68.1 \pm 7.5$ & $75.5 \pm 8.3$ & $79.3 \pm 3.7$ \\
{$[\mathbf{4 - 2 ]}$} & & $73.8 \pm 9.5$ & $77.1 \pm 5.8$ & $78.8 \pm 3.6$ & $67.4 \pm 6.6$ & $69.3 \pm 5.5$ & $71.5 \pm 5.7$ \\
{$[\mathbf{4 - 3}]$} & & $70.7 \pm 5.4$ & $74.7 \pm 8.2$ & $75.2 \pm 2.3$ & $65.8 \pm 9.1$ & $69.7 \pm 5.7$ & $70.9 \pm 1.1$ \\
{$[\mathbf{3 - 0}]$} & 50.0 & $69.9 \pm 2.0$ & $71.6 \pm 5.5$ & $79.3 \pm 3.7$ & $69.5 \pm 11.5$ & $73.3 \pm 5.0$ & $78.8 \pm 3.6$ \\
{$[\mathbf{3 - 1}]$} & & $68.7 \pm 7.5$ & $75.0 \pm 9.1$ & $77.7 \pm 7.8$ & $58.3 \pm 9.1$ & $59.3 \pm 2.3$ & $64.2 \pm 11.0$ \\
{$[\mathbf{3 - 2}]$} & & $65.1 \pm 7.9$ & $71.9 \pm 6.5$ & $72.3 \pm 4.6$ & $60.6 \pm 6.5$ & $71.3 \pm 7.8$ & $71.9 \pm 6.5$ \\
{$[\mathbf{2 - 0}]$} & & $78.8 \pm 9.5$ & $79.3 \pm 3.3$ & $81.2 \pm 5.5$ & $61.5 \pm 2.9$ & $68.3 \pm 5.6$ & $73.8 \pm 9.5$ \\
{$[\mathbf{2 - 1}]$} & & $77.1 \pm 6.8$ & $75.8 \pm 5.0$ & $79.6 \pm 7.7$ & $63.1 \pm 10.5$ & $74.1 \pm 1.5$ & $77.1 \pm 5.8$ \\
{$[\mathbf{1 - 0}]$} & & $59.6 \pm 8.5$ & $69.5 \pm 4.7$ & $69.4 \pm 5.1$ & $59.9 \pm 3.4$ & $69.5 \pm 4.1$ & $68.1 \pm 7.5$ \\
\hline$[$ all-all] & 20.0 & $39.8 \pm 4.7$ & $41.7 \pm 5.6$ & $42.2 \pm 5.5$ & $38.0 \pm 4.6$ & $40.3 \pm 6.2$ & $41.2 \pm 4.0$ \\
\hline
\end{tabular}

investigate whether the position of the attention would have a certain impact on the results. There are five alternative positions in our architecture (Fig. 3), which are located after four convolution layers in the shared block and one convolution layer in the dual-branch. These positions are numbered Position $=1,2, \ldots, 5$ in order. We try to put the attention in these different positions. The results are shown in Fig. 7. From these results, we could know high-level semantic features may not be suitable for attention structures. The reason may be that the receptive field of these features are not large enough, and the related pain regions are not yet involved. The low-level features' receptive fields are too wide to focus. Besides, multiple attention structures are not conducted in this experiment. This is because the results of some positions are inferior to those without attention and multiple attention structures may bring the risk of overfitting, which are also indicated in [51]. Thus, the appropriate attention structure facilitates the proposed pain estimation network to focus on more important information.

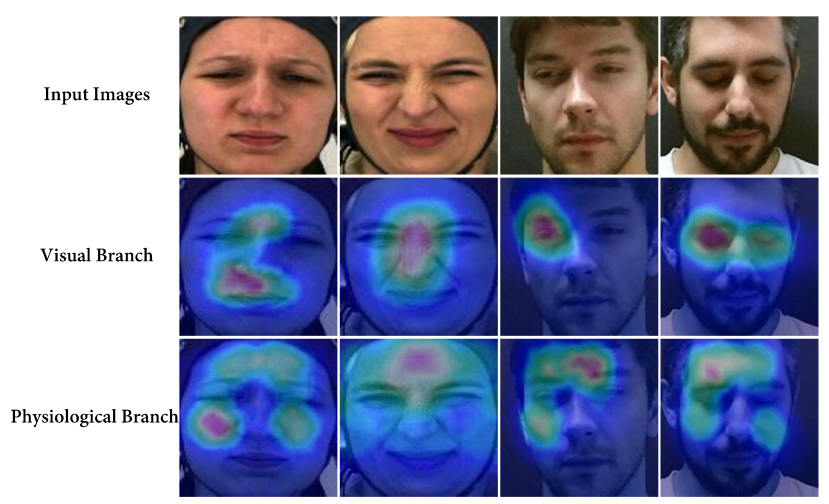

Fig. 8. The attention maps of visual and physiological branches.

3) The Evaluation of Dual Branches: To evaluate the collaboration of two branches, the deep models with various structures employing only one branch are compared. Table III shows the comparison results between different models. As can be seen, the structure of dual branches performs best on both datasets. This suggests the using of visual and physiological branches allows the model to consider a more comprehensive representation of pain. Besides, observed from the comparison of visual and physiological branches, the task of pain estimation can obtain more useful information from visual facial pain features while the pain estimation task could also benefit from the pseudo physiological modality.

TABLE III

The Evaluation of Dual Branches.

\begin{tabular}{cccc}
\hline Dataset & Structure & Accuracy[4-0] & Accuracy[all-all] \\
\hline \multirow{3}{*}{ Biovid } & Visual Branch & $77.5 \pm 7.6$ & $34.3 \pm 4.8$ \\
& Physiological Branch & $65.0 \pm 4.2$ & $28.5 \pm 2.5$ \\
& Dual Branches & $88.1 \pm 9.3$ & $42.2 \pm 5.5$ \\
\hline \multirow{2}{*}{ Mint } & Visual Branch & $65.8 \pm 6.2$ & $28.5 \pm 3.0$ \\
& Physiological Branch & $55.5 \pm 7.1$ & $22.6 \pm 3.6$ \\
& Dual Branches & $80.9 \pm 5.6$ & $41.2 \pm 4.0$ \\
\hline
\end{tabular}

In addition, the proposed dual branches partially share some blocks. We also test the fully shared situation, that is, all feature layers are shared. The results are shown in Fig. 9. As can be seen, the fully shared structure does not work well for our setting. This suggests the focuses of facial pain and pseudo physiological features are not exactly the same regions. While they may have some related connection in low-level features, there are some differences in the attention layers. In order to verify our ideas, we also visualize the attention map of the physiological branch, as shown in third row of Fig. 8. The comparison with the visual branch attention maps shows that although these tasks are related, their manifestations of important information are different. So we devise the dual branches for visual pain estimation and physiological HRG estimation. This way could not only explore the inner connection between these tasks but also adapt to the uniqueness of individual task.

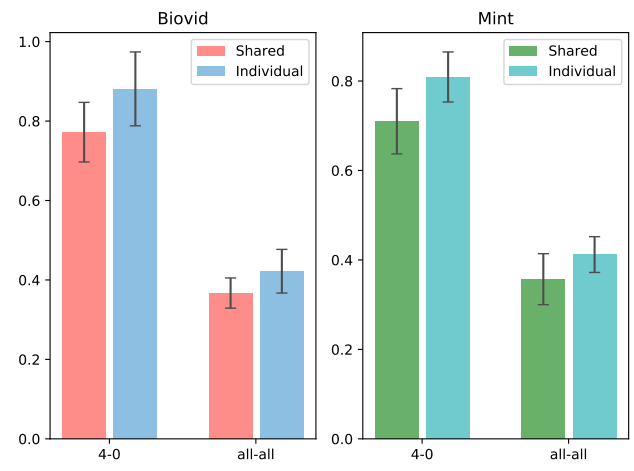

Fig. 9. The comparison between shared network and individual network. 
4) The Estimation of the HRG Analyzer: This paper classifies pain into five categories on two datasets. The HRG analyzer is used to generate the probability of HRG at each pain intensity. In this section, we employ the proposed HRG Analyzer to quantify $H R G$ for pain estimation. The $H R G$ distribution of each pain intensity is described according to the HRG results in two training sets, as shown in Figs. 10 and 11. We summarize these distribution curves into graphs, as shown in the Figs. 12 and 13. In Fig. 12, the curve of $P I=2$ is close to that of other pain intensities. The reason is that pain in the middle intensity is not distinguishable enough from the pain in adjacent intensities. But when the pain intensity differs by more than two levels, the distinction is considerable. In general, when pain is severer, the HRG value is more concentrated on the distribution in larger values. The reason is that pain is not only reflected in the movements of face, it also causes relatively large variance in physiological signals. When an individual feels pain, the heart rate increases correspondingly. The severer the pain, the more heart rate increases.

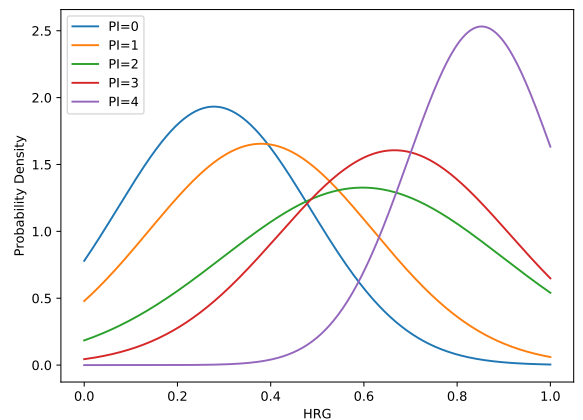

Fig. 12. The distribution of HRG under different pain intensities on Biovid dataset.

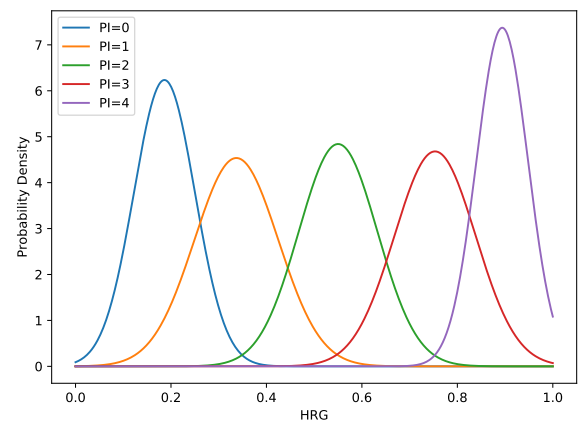

Fig. 13. The distribution of HRG under different pain intensities on Mint dataset.

In order to explain our analyzer clearly, we employ the Bayesian formula to obtain the probability of each pain level from the analyzer when the HRG values are 0.2 and 0.8 , respectively, as shown in Fig. 14. Observed from the results of two datasets, when the HRG becomes larger, the probabilities of high pain intensities become larger in analyzer results. In this paper, these probabilities allow us to estimate pain intensity from another perspective.

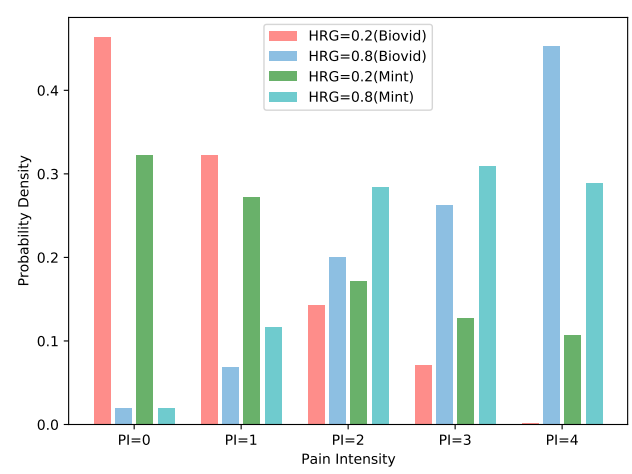

Fig. 14. The probabilities of pain intensities under various HRGs on two datasets.

5) The Evaluation of Fusion Strategy: We compare the results under different fusion strategies: without fusion, feature fusion, decision fusion and the proposed probability fusion. The "without fusion" denotes the results are from the visual branch. For the feature fusion, we implement it by concatenating the last feature layers of two branches and train the model directly. For the decision fusion, after the separate training phase, we modify the output layer of the physiological branch for pain intensity estimation, and combine this output layer with that of the visual branch for jointly training. These results are shown in Fig. 15. Observed from this figure, the HRG analyzer could significantly improve the performance of pain estimation. This suggests that it is feasible to find clues for pain estimation from heart rate changes. In addition, from the results of feature fusion and decision fusion, there is not much difference between these two strategies, but they are better than the strategy without fusion. This also illustrates that comprehensive-perspective consideration leads to a more accurate pain estimation performance. In conclusion, these results suggest that the proposed HRG analyzer could map the HRG to the pain intensities probabilities. This method provides a worthy reference for our non-contact method that finds new solutions for pain estimation research from pseudo physiological signals.

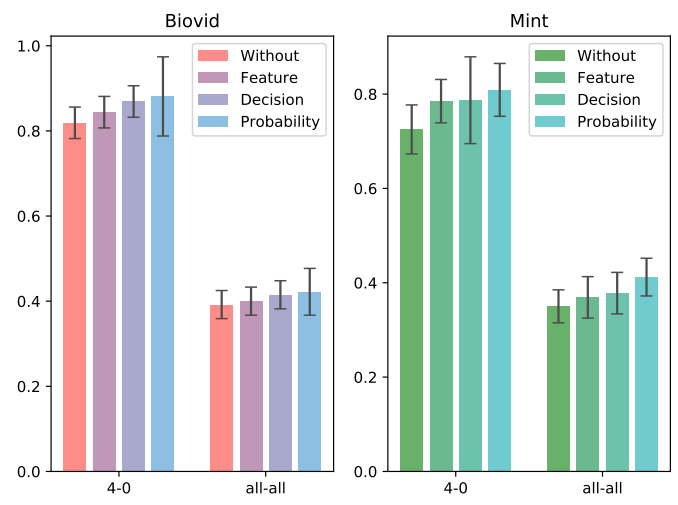

Fig. 15. The comparison of different fusion strategies. The 'Without', 'Feature', 'Decision' and 'Probability' mean without fusion, feature fusion, decision fusion and probability fusion, respectively. 

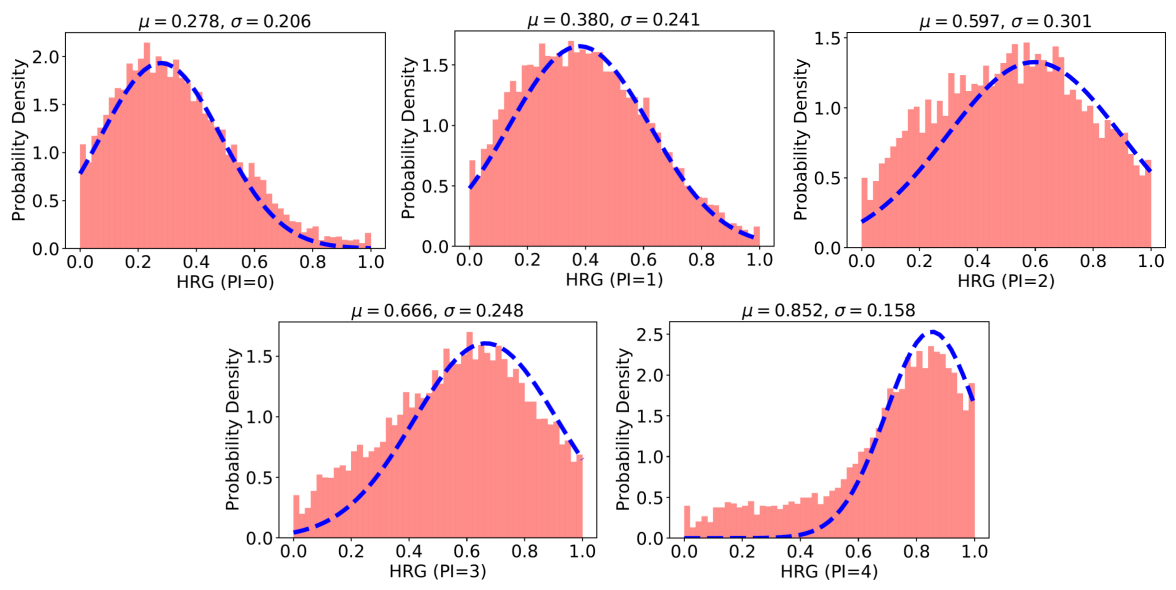

Fig. 10. The histograms of HRG under different pain intensities on Biovid dataset.
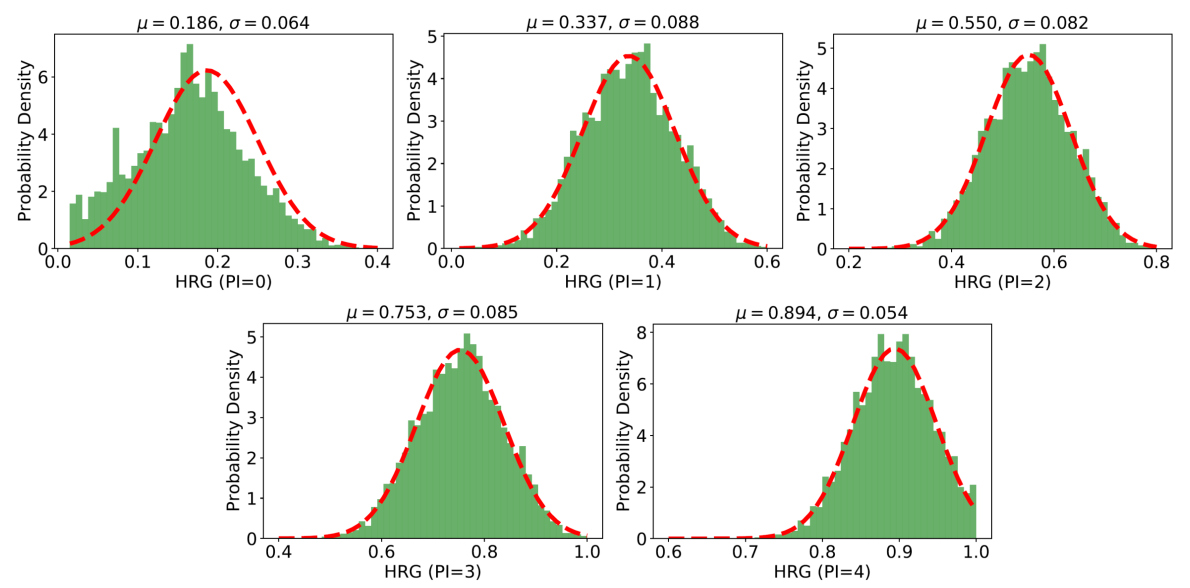

Fig. 11. The histograms of HRG under different pain intensities on Mint dataset.

\section{Comparison with Existing Methods}

In this section, we compare the proposed method against other existing methods. We begin this by comparing some multi-modal methods on the Biovid dataset, which provides the physiological signals measured by physical contact devices. This comparison is shown in Table IV. These methods analyze pain information from multi-modal signals. They process the collected physiological signals and video signals accordingly. Some focused ECG, EMG and GSR (e.g., [16], [18], [34], [35]) and others integrate video signals, (e.g., [16], [17]). They obtained these signals through some signal collectors connected to the human body, and then comprehensively analyzed the relationship between these signals and pain. Although they achieved good experimental results, it was difficult to promote their applications due to the limitations of signal acquisition methods.

To ascertain whether the performance between the proposed method and other methods are statistically meaningful, the two-sample T-test is performed between accuracy values obtained by different methods. The confidence threshold is set to 0.95 . Compared with others, the proposed method has an obvious statistical superiority with only two exceptions, where our method has similar performance. This is because our
TABLE IV

COMPARISON WITH THE MULTI-MODAL METHODS ON BIOVID DATASET.

\begin{tabular}{ccc}
\hline Methods & Accuracy[4-0] & Accuracy[all-all] \\
\hline Early Fusion (MP) [16] & $75.6^{*}$ & N/A \\
Early Fusion (MP\&V) [16] & $80.6^{*}$ & N/A \\
Logistic Regression (MP) [18] & $74.2 \pm 17.5^{*}$ & N/A \\
Early Fusion (MP\&V) [17] & $82.7^{*}$ & N/A \\
Semi-supervised Learning (MP) [34] & 85.7 & 40.1 \\
Concatenated Representation (MP) [35] & $77.2 \pm 17.5^{*}$ & N/A \\
Shared Representation (MP) [35] & $76.9 \pm 15.1^{*}$ & N/A \\
Gated Representation (MP) [35] & $84.0 \pm 15.6$ & N/A \\
Our Proposed (V) & $\mathbf{8 8 . 1} \pm \mathbf{9 . 3}$ & $\mathbf{4 2 . 2} \pm \mathbf{5 . 5}$ \\
\hline
\end{tabular}

'MP' and ' $\mathrm{V}$ ' mean this method involves multiple physiological signals and video signals, respectively.

Significantly worse $(p<0.05)$ than our proposed method.

method employs the advanced spatio-temporal decomposition of the deep residual network, which has a stronger ability to express pain intensity than the multi-modal method using hand-crafted statistical features.

Next, we compare our method with other visual methods, and the comparison on the Biovid dataset is shown in Table $\mathrm{V}$. These methods only use video sequences as input. In the early years, most of the research used hand-crafted features [11], [52], and later moved to deep network methods [28], 
TABLE V

COMPARISON WITH THE VISUAL METHODS ON BIOVID DATASET.

\begin{tabular}{ccc}
\hline Methods & Accuracy[4-0] & Accuracy[all-all] \\
\hline Facial 3D Distances [11] & $72.1^{*}$ & $30.3^{*}$ \\
Facial Activity Descriptors [11] & $72.4^{*}$ & $30.8^{*}$ \\
Optical Flow Method [52] & $70.2^{*}$ & N/A \\
Spatiotemporal CNN [28] & 86.0 & N/A \\
Self-supervised Distillation [53] & $71.0^{*}$ & N/A \\
Pain-awareness Network [54] & $71.3 \pm 11.6^{*}$ & $37.6 \pm 9.2 *$ \\
Spatio-Temporal Attention Network [51] ${ }^{\dagger}$ & $73.9 \pm 8.8^{*}$ & $39.1 \pm 10.4$ \\
Our Proposed & $\mathbf{8 8 . 1} \pm \mathbf{9 . 3}$ & $\mathbf{4 2 . 2} \pm \mathbf{5 . 5}$ \\
\hline
\end{tabular}

$\dagger$ This method is reimplemented for pain intensity estimation on Biovid dataset.

* Significantly worse $(p<0.05)$ than our proposed method.

[53], [51]. But compared with the previous table (Table IV), we could roughly observe that the results of these methods are mostly worse than the multi-modal results. This suggests that these contactless visual methods only consider how to find the pain information from video texture. They have never jumped out of this valley, thinking from other points. Therefore, we try to obtain the information that helps to estimate pain from other physiological signals. This is the strongest motivation for our work. On the one hand, we extract the spatio-temporal dynamic pain texture features from the video. On the other hand, we estimate the pseudo physiological signal from videos so that it affects the results of pain estimation. Then we could estimate the pain intensity from the perspectives of both facial expressions and physiological signals, so that we achieved better results.

TABLE VI

COMPARISON WITH THE VISUAL METHODS ON Mint DATASET.

\begin{tabular}{ccc}
\hline Methods & Accuracy[4-0] & Accuracy[all-all] \\
\hline VGG-Face CNN with LSTM [48] $^{\dagger}$ & N/A & $36.6^{*}$ \\
Spatio-Temporal Feature Gating [55] & $72.9 \pm 8.7^{*}$ & $38.6 \pm 5.7^{*}$ \\
Convolutional 3D Network [49] $^{\dagger}$ & $71.2 \pm 3.3^{*}$ & $38.0 \pm 4.6^{*}$ \\
3D Residual ConvNet [50] & $74.6 \pm 2.4^{*}$ & $40.3 \pm 6.2$ \\
Pain-awareness Network [54] $^{\dagger}$ & $68.8 \pm 9.3^{*}$ & $35.4 \pm 4.0^{*}$ \\
Spatio-Temporal Attention Network [51] $^{\dagger}$ & $75.4 \pm 6.7^{*}$ & $38.1 \pm 4.1^{*}$ \\
Visual Branch & $65.8 \pm 6.2^{*}$ & $28.5 \pm 3.0^{*}$ \\
Our Proposed & $\mathbf{8 0 . 9} \pm \mathbf{5 . 6}$ & $\mathbf{4 1 . 2} \pm \mathbf{4 . 0}$
\end{tabular}

\footnotetext{
${ }^{\dagger}$ This method is reimplemented for pain intensity estimation on Mint dataset

Significantly worse $(p<0.05)$ than our proposed method.
}

Besides, we compare the results of some methods on Mint dataset. Because this dataset was released recently, there is less research on this dataset. And it does not provided physiological signals, we reimplemented some representative visual methods used in action recognition for the comparison. This comparison is shown in Table VI. Observed from the results, the experimental results on this dataset are obviously inferior to that of Biovid dataset. The reason is that Mint dataset was collected in a less-constrained environment, so it is more challenging. This could be observed from Fig. 5. But it provides the opportunity to test the methods with data closer to real world scenario, even though the results are not as good as from Biovid as expected. And from the results comparison, our method still achieves better performance due to its ability to estimate pain from multiple perspectives. In the significance test, most results are worse than ours.

\section{CONCLUSiOn AND Future Work}

In this paper, we proposed a deep spatio-temporal pain estimation network by measuring the HRG value that reflects the relative variation to the signal in the painless condition. Firstly, some preprocessing techniques including face cropping, key frames selection and data augmentation were employed for training the deep model. Then, a dual-branch pain estimation network was devised to employ R(2+1)D structure and attention structure to extract visual and physiological features for pain estimation by generating pseudo HRG values, respectively. For the HRG values, we presented an analyzer that leverages the quantitative relationship of pain intensity and HRG value. Finally, extensive evaluation experiments were implemented on two publicly-available datasets. The experimental results showed the proposed method achieves promising performance.

In future work, we will continue to estimate other physiological signals from videos for improving pain estimation performance, such as blood pressure and pulse. Intuitively, these physiological signals also change when people feel pain. If we utilize a similar method to create some quantitative mapping relationships, we could obtain a more complete pain estimation system for exploiting contactless multi-modal information.

\section{ACKNOWLEDGMENT}

We would like to thank the editor, reviewers and Mr. Jonathan Fortune for improving the quality of this paper. This work is partly supported by the Key Research and Development Program of Shaanxi (Program Nos. 2021ZDLGY1501, 2021ZDLGY09-04, 2021GY-004 and 2020GY-050), the National Natural Science Foundation of China (Grant No. 61772419), Academy of Finland for project MiGA (Grant No. 316765), and ICT 2023 project (Grant No. 328115), Infotech Oulu.

\section{REFERENCES}

[1] H. Merskey, D. Albe-Fessard, J. Bonica, A. Carmen, R. Dubner, F. Kerr and C. Pagni, "Editorial: The need of a taxonomy," Pain, vol. 6, no. 3, pp. 247-252, 1979.

[2] M. J. Sullivan, P. Thibault, A. Savard, R. Catchlove, J. Kozey, and W. D. Stanish, "The influence of communication goals and physical demands on different dimensions of pain behavior," Pain, vol. 125, no. 3, pp. 270-277, 2006.

[3] P. Mäntyselkä, E. Kumpusalo, R. Ahonen, A. Kumpusalo, J. Kauhanen, H. Viinamäki, P. Halonen, and J. Takala, "Pain as a reason to visit the doctor: a study in finnish primary health care," Pain, vol. 89, no. 2-3, pp. 175-180, 2001.

[4] M. Von Korff, M. P. Jensen, and P. Karoly, "Assessing global pain severity by self-report in clinical and health services research," Spine, vol. 25 , no. 24, pp. 3140-3151, 2000.

[5] S. M. Zwakhalen, J. P. Hamers, H. H. Abu-Saad, and M. P. Berger, "Pain in elderly people with severe dementia: a systematic review of behavioural pain assessment tools," BMC Geriatrics, vol. 6, no. 1, p. 3 , 2006.

[6] G. C. Littlewort, M. S. Bartlett, and K. Lee, "Automatic coding of facial expressions displayed during posed and genuine pain," Image and Vision Computing, vol. 27, no. 12, pp. 1797-1803, 2009.

[7] A. B. Ashraf, S. Lucey, J. F. Cohn, T. Chen, Z. Ambadar, K. M. Prkachin, and P. E. Solomon, "The painful face-pain expression recognition using active appearance models," Image and Vision Computing, vol. 27, no. 12, pp. 1788-1796, 2009. 
[8] C. Florea, L. Florea, and C. Vertan, "Learning pain from emotion: transferred hot data representation for pain intensity estimation," in European Conference on Computer Vision (ECCV). Springer, 2014, pp. $778-790$.

[9] F. Wang, X. Xiang, C. Liu, T. D. Tran, A. Reiter, G. D. Hager, H. Quon, J. Cheng, and A. L. Yuille, "Regularizing face verification nets for pain intensity regression," in IEEE International Conference on Image Processing (ICIP). IEEE, 2017, pp. 1087-1091.

[10] R. Yang, X. Hong, J. Peng, X. Feng, and G. Zhao, "Incorporating high-level and low-level cues for pain intensity estimation," in 24th International Conference on Pattern Recognition (ICPR). IEEE, 2018, pp. 3495-3500.

[11] P. Werner, A. Al-Hamadi, K. Limbrecht-Ecklundt, S. Walter, S. Gruss, and H. C. Traue, "Automatic pain assessment with facial activity descriptors," IEEE Transactions on Affective Computing, vol. 8, no. 3, pp. 286-299, 2016.

[12] D. Liu, F. Peng, A. Shea, and R. Picard, "Deepfacelift: Interpretable personalized models for automatic estimation of self-reported pain," Journal of Machine Learning Research, vol. 66, pp. 1-16, 2017.

[13] J. Zhou, X. Hong, F. Su, and G. Zhao, "Recurrent convolutional neural network regression for continuous pain intensity estimation in video," in Proceedings of the IEEE Conference on Computer Vision and Pattern Recognition Workshops, 2016, pp. 84-92.

[14] P. Rodriguez, G. Cucurull, J. Gonzàlez, J. M. Gonfaus, K. Nasrollahi, T. B. Moeslund, and F. X. Roca, "Deep pain: Exploiting long short-term memory networks for facial expression classification," IEEE Transactions on Cybernetics, 2017.

[15] M. Tavakolian and A. Hadid, "Deep binary representation of facial expressions: A novel framework for automatic pain intensity recognition," in 25th IEEE International Conference on Image Processing (ICIP). IEEE, 2018, pp. 1952-1956.

[16] P. Werner, A. Al-Hamadi, R. Niese, S. Walter, S. Gruss, and H. C. Traue, "Automatic pain recognition from video and biomedical signals," in 22nd International Conference on Pattern Recognition (ICPR). IEEE, 2014, pp. $4582-4587$.

[17] M. Kächele, P. Werner, A. Al-Hamadi, G. Palm, S. Walter, and F. Schwenker, "Bio-visual fusion for person-independent recognition of pain intensity," in International Workshop on Multiple Classifier Systems. Springer, 2015, pp. 220-230.

[18] D. Lopez-Martinez and R. Picard, "Multi-task neural networks for personalized pain recognition from physiological signals," in Seventh International Conference on Affective Computing and Intelligent Interaction Workshops and Demos (ACIIW). IEEE, 2017, pp. 181-184.

[19] Z. Yu, W. Peng, X. Li, X. Hong, and G. Zhao, "Remote heart rate measurement from highly compressed facial videos: An end-to-end deep learning solution with video enhancement," in IEEE/CVF International Conference on Computer Vision (ICCV), 2019, pp. 151-160.

[20] X. Niu, Z. Yu, H. Han, X. Li, S. Shan, and G. Zhao, "Video-based remote physiological measurement via cross-verified feature disentangling," arXiv preprint arXiv:2007.08213, 2020.

[21] A. J. Terkelsen, H. Mølgaard, J. Hansen, O. K. Andersen, and T. S. Jensen, "Acute pain increases heart rate: differential mechanisms during rest and mental stress," Autonomic Neuroscience, vol. 121, no. 1-2, pp. 101-109, 2005.

[22] B. M. Appelhans and L. J. Luecken, "Heart rate variability and pain: associations of two interrelated homeostatic processes," Biological Psychology, vol. 77, no. 2, pp. 174-182, 2008.

[23] P. Lucey, J. F. Cohn, K. M. Prkachin, P. E. Solomon, S. Chew, and I. Matthews, "Painful monitoring: Automatic pain monitoring using the unbc-mcmaster shoulder pain expression archive database," Image and Vision Computing, vol. 30, no. 3, pp. 197-205, 2012.

[24] O. Rudovic, V. Pavlovic, and M. Pantic, "Context-sensitive dynamic ordinal regression for intensity estimation of facial action units," IEEE Transactions on Pattern Analysis and Machine Intelligence, vol. 37, no. 5, pp. 944-958, 2014.

[25] R. Irani, K. Nasrollahi, and T. B. Moeslund, "Pain recognition using spatiotemporal oriented energy of facial muscles," in Proceedings of the IEEE Conference on Computer Vision and Pattern Recognition Workshops, 2015, pp. 80-87.

[26] R. Zhao, Q. Gan, S. Wang, and Q. Ji, "Facial expression intensity estimation using ordinal information," in Proceedings of the IEEE Conference on Computer Vision and Pattern Recognition (CVPR), 2016, pp. 3466-3474.

[27] L. Martinez, D. Rosalind Picard et al., "Personalized automatic estimation of self-reported pain intensity from facial expressions," in Proceedings of the IEEE Conference on Computer Vision and Pattern Recognition Workshops, 2017, pp. 70-79.
[28] M. Tavakolian and A. Hadid, "A spatiotemporal convolutional neural network for automatic pain intensity estimation from facial dynamics," International Journal of Computer Vision, pp. 1-13, 2019.

[29] P. Tzirakis, G. Trigeorgis, M. A. Nicolaou, B. W. Schuller, and S. Zafeiriou, "End-to-end multimodal emotion recognition using deep neural networks," IEEE Journal of Selected Topics in Signal Processing, vol. 11, no. 8, pp. 1301-1309, 2017.

[30] C.-Y. Chang, J.-S. Tsai, C.-J. Wang, and P.-C. Chung, "Emotion recognition with consideration of facial expression and physiological signals," in IEEE Symposium on Computational Intelligence in Bioinformatics and Computational Biology. IEEE, 2009, pp. 278-283.

[31] W. Zheng, W. Liu, Y. Lu, B. Lu, and A. Cichocki, "Emotionmeter: A multimodal framework for recognizing human emotions," IEEE Transactions on Cybernetics, vol. 49, no. 3, pp. 1110-1122, 2019.

[32] Y. Chu, X. Zhao, J. Han, and Y. Su, "Physiological signal-based method for measurement of pain intensity," Frontiers in neuroscience, vol. 11, p. 279, 2017.

[33] Z. Hammal and M. Kunz, "Pain monitoring: A dynamic and contextsensitive system," Pattern Recognition, vol. 45, no. 4, pp. 1265-1280, 2012.

[34] M. Kächele, P. Thiam, M. Amirian, F. Schwenker, and G. Palm, "Methods for person-centered continuous pain intensity assessment from bio-physiological channels," IEEE Journal of Selected Topics in Signal Processing, vol. 10, no. 5, pp. 854-864, 2016

[35] P. Thiam, H. A. Kestler, and F. Schwenker, "Multimodal deep denoising convolutional autoencoders for pain intensity classification based on physiological signals." in ICPRAM, 2020, pp. 289-296.

[36] X. Li, J. Chen, G. Zhao, and M. Pietikainen, "Remote heart rate measurement from face videos under realistic situations," in Proceedings of the IEEE Conference on Computer Vision and Pattern Recognition (CVPR), 2014, pp. 4264-4271.

[37] T. Baltrusaitis, A. Zadeh, Y. C. Lim, and L. Morency, "Openface 2.0: Facial behavior analysis toolkit," in 13th IEEE International Conference on Automatic Face Gesture Recognition (FG), 2018, pp. 59-66.

[38] M. S. Hossain and G. Muhammad, "Emotion recognition using deep learning approach from audio-visual emotional big data," Information Fusion, vol. 49, pp. 69-78, 2019.

[39] Z. Xia, X. Hong, X. Gao, X. Feng, and G. Zhao, "Spatiotemporal recurrent convolutional networks for recognizing spontaneous microexpressions," IEEE Transactions on Multimedia, vol. 22, no. 3, pp. 626640, 2020.

[40] K. Kumar and D. D. Shrimankar, "F-des: Fast and deep event summarization," IEEE Transactions on Multimedia, vol. 20, no. 2, pp. 323-334, 2018.

[41] D. Tran, H. Wang, L. Torresani, J. Ray, Y. LeCun, and M. Paluri, "A closer look at spatiotemporal convolutions for action recognition," in Proceedings of the IEEE conference on Computer Vision and Pattern Recognition (CVPR), 2018, pp. 6450-6459.

[42] J. Li, X. Liu, W. Zhang, M. Zhang, J. Song, and N. Sebe, "Spatiotemporal attention networks for action recognition and detection," IEEE Transactions on Multimedia, vol. 22, no. 11, pp. 2990-3001, 2020.

[43] C. Yan, Y. Tu, X. Wang, Y. Zhang, X. Hao, Y. Zhang, and Q. Dai, "Stat: Spatial-temporal attention mechanism for video captioning," IEEE Transactions on Multimedia, vol. 22, no. 1, pp. 229-241, 2020.

[44] M. A. Hassan, A. S. Malik, D. Fofi, N. M. Saad, B. Karasfi, Y. S. Ali, and F. Meriaudeau, "Heart rate estimation using facial video: A review," Biomedical Signal Processing and Control, vol. 38, pp. 346-360, 2017.

[45] K. M. Prkachin and P. E. Solomon, "The structure, reliability and validity of pain expression: Evidence from patients with shoulder pain," Pain vol. 139, no. 2, pp. 267-274, 2008.

[46] Y. Peng, J. Qi, X. Huang, and Y. Yuan, "Ccl: Cross-modal correlation learning with multigrained fusion by hierarchical network," IEEE Transactions on Multimedia, vol. 20, no. 2, pp. 405-420, 2018.

[47] S. Walter, S. Gruss, H. Ehleiter, J. Tan, H. C. Traue, P. Werner, A. AlHamadi, S. Crawcour, A. O. Andrade, and G. M. da Silva, "The biovid heat pain database data for the advancement and systematic validation of an automated pain recognition system," in IEEE International Conference on Cybernetics. IEEE, 2013, pp. 128-131.

[48] M. A. Haque, R. B. Bautista, F. Noroozi, K. Kulkarni, C. B. Laursen, R. Irani, M. Bellantonio, S. Escalera, G. Anbarjafari, K. Nasrollahi, O. K. Andersen, E. G. Spaich, and T. B. Moeslund, "Deep multimodal pain recognition: A database and comparison of spatio-temporal visual modalities," in 13th IEEE International Conference on Automatic Face Gesture Recognition (FG). IEEE, 2018, pp. 250-257.

[49] D. Tran, L. Bourdev, R. Fergus, L. Torresani, and M. Paluri, "Learning spatiotemporal features with $3 \mathrm{~d}$ convolutional networks," in IEEE International Conference on Computer Vision (ICCV), 2015, pp. 4489-4497. 
[50] D. Tran, J. Ray, Z. Shou, S.-F. Chang, and M. Paluri, "Convnet architecture search for spatiotemporal feature learning," arXiv preprint arXiv:1708.05038, 2017.

[51] D. Huang, Z. Xia, J. Mwesigye, and X. Feng, "Pain-attentive network: a deep spatio-temporal attention model for pain estimation," Multimedia Tools and Applications, vol. 79, no. 37, pp. 28 329-28 354, 2020.

[52] P. Werner, A. Al-Hamadi, and S. Walter, "Analysis of facial expressiveness during experimentally induced heat pain," in Seventh International Conference on Affective Computing and Intelligent Interaction Workshops and Demos (ACIIW). IEEE, 2017, pp. 176-180.

[53] M. Tavakolian, M. B. Lopez, and L. Liu, "Self-supervised pain intensity estimation from facial videos via statistical spatiotemporal distillation," Pattern Recognition Letters, 2020.

[54] D. Huang, Z. Xia, L. Li, K. Wang, and X. Feng, "Pain-awareness multistream convolutional neural network for pain estimation," Journal of Electronic Imaging, vol. 28, no. 4, p. 043008, 2019.

[55] S. Xie, C. Sun, J. Huang, Z. Tu, and K. Murphy, "Rethinking spatiotemporal feature learning: Speed-accuracy trade-offs in video classification," in European Conference on Computer Vision (ECCV). Springer, 2018, pp. 318-335.

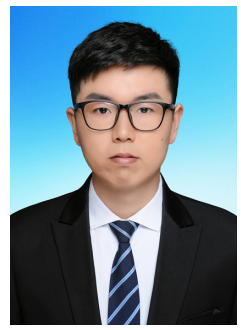

Dong Huang received his M.S. degree from the School of Electronics and Information, Northwestern Polytechnical University, China, in 2017. He is currently pursuing his Ph.D. in Northwestern Polytechnical University. His current research interests include machine learning, deep learning, attention models and expression recognition.

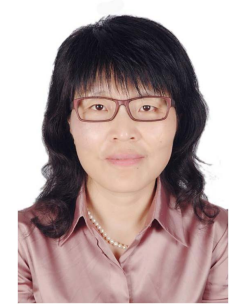

Xiaoyi Feng received the M.S. degree from Northwest University, Xian, China, in 1994, and the Ph.D. degree from Northwestern Polytechnical University, Xian, in 2001. She has been a Professor with the School of Electronics and Information, Northwestern Polytechnical University, since 2008. She has authored or coauthored more than 80 papers in journals and conferences. Her current research interests include computer vision, image process, radar imagery, and recognition.

Haixi Zhang received the M.S. degree and PHD degree from Northwestern Polytechnical University, Xian, China, in 2013 and 2020, respectively. He is working at College of Information Engineering, Northwest A\&F University, Shaanxi, China. His research interests include machine learning, computer vision and image processing.

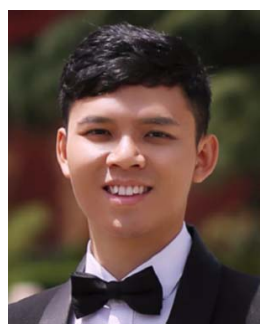

Zitong Yu (Graduate Student Member, IEEE) received the M.S. degree from the University of Nantes, France, in 2016. He is currently pursuing the Ph.D. degree with the Center for Machine Vision and Signal Analysis, University of Oulu, Finland. His research interests focus on remote physiological measurement, face anti-spoofing, and video understanding.

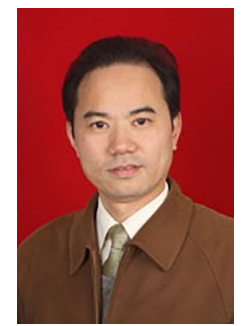

Jinye Peng is a Professor with the Northwest University of China. He received his Ph.D. degree from Northwestern Polytechnical University of China. His research interests include computer version, pattern recognition and signal processing.

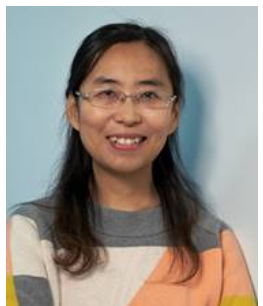

Guoying Zhao (Senior Member, IEEE) received the $\mathrm{Ph} . \mathrm{D}$. degree in computer science from the Chinese Academy of Sciences, Beijing, China, in 2005. She is currently a Professor with the Center for Machine Vision and Signal Analysis, University of Oulu, Finland, where she has been a Senior Researcher since 2005, and an Associate Professor since 2014. She has authored or coauthored more than 240 papers in journals and conferences. Her papers have currently over 14500 citations in Google Scholar (H-index 55). She has served as a area chairs for several conferences and is an Associate Editor for Pattern Recognition, IEEE Transactions on Circuits and Systems for Video Technology, and Image and Vision Computing journals. She is a Fellow of IAPR.

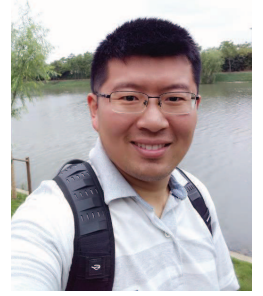

Zhaoqiang Xia (Member, IEEE) received the B.E. degree and Ph.D. degree from the Northwestern Polytechnical University, Xi' an, China, in 2008 and 2014, respectively. He has been a visiting scholar with the University of North Carolina at Charlotte from 2011 to 2013 and a senior researcher with the University of Oulu in 2019. He is currently an associate professor in the School of Electronics and Information, Northwestern Polytechnical University He has authored or co-authored more than 60 papers in international journals and conferences, and has served as a reviewer for mainstream journals and session co-chairs for international conferences. His current research interests include image processing, visual search and recognition, and statistical machine learning. 